\title{
Die Sonderverwaltungszone Hong Kong und das "Common Law" \\ Zur Anwendbarkeit des "Common Law" in Hong Kong nach dem Souveränitätswechsel ${ }^{1}$
}

\author{
Von Tim G. Luthra
}

\section{Einleitung}

Seit dem 1. Juli 1997 übt die Volksrepublik China die vollständige Souveränität über die Sonderverwaltungszone Hong Kong aus, nachdem Hong Kong zuvor über einen Zeitraum von mehr als 150 Jahren Kronkolonie des Vereinigten Königreichs ${ }^{2}$ war. $^{3}$ Die Entwicklung, an deren Ende die "Rückgabe" der Kronkolonie Hong Kong durch das Vereinigte Königreich an die Volksrepublik China stand, soll im folgenden kurz skizziert werden, wobei darauf hinzuweisen ist, daß die Volksrepublik China und das Vereinigte Königreich stets unterschiedliche Auffassungen zum rechtlichen Status von Hong Kong vertreten haben, ohne daß es im Rahmen dieses Beitrages möglich wäre, die divergierenden Standpunkte im einzelnen darzustellen. ${ }^{4}$

Die Kolonialisierung von Hong Kong erfolgte im wesentlichen in drei Etappen, wobei die Insel Hong Kong und ein Stück des chinesischen Festlandes mit der Bezeichnung Kowloon

Ganz herzlichen Dank schulde ich Frau Jennifer M. Y. Wu-Scharsig, LL.M., als Solicitor zugelassen in Hong Kong, England und Wales, für ihre vielfältige Hilfe bei der Bearbeitung dieses Aufsatzes.

2

Vereinigtes Königreich von Großbritannien und Nordirland (nachfolgend: "Vereinigtes Königreich").

Zur Abtretung der Gebiete Hong Kong Island und Kowloon sowie zum Pachtvertrag über die "New Territories" sogleich und Scheuer, M., Die Rechtslage von Hong Kong und Macao nach den "Gemeinsamen Erklärungen" vom 19. Dezember 1984 und 13. April 1987, Frankfurt a. M. 1993, S. $24 \mathrm{ff}$.

4

Die Beurteilung der Entwicklung bis zur Rückübertragung von Hong Kong am 1. Juli 1997 folgt der überwiegenden Auffassung im westlichen Schrifttum, die von einer rechtlich wirksamen Abtretung der Gebiete Hong Kong Island und Kowloon an das Vereinigte Königreich ausgeht. Zu den unterschiedlichen Auffassungen vgl. Hughes, A., Hong Kong, in: Bernhardt, R. (Hrsg.), Encyclopedia of Public International Law, Vol. II, Amsterdam / Lausanne / New York / Oxford / Shannon / Tokyo 1995, S. 870 ff.; Scheuer, M., Die Rechtslage von Hong Kong und Macao nach den "Gemeinsamen Erklärungen" vom 19. Dezember 1984 und 13. April 1987, Fn. 3, S. 24 ff. und Yash Ghai, Hong Kong's New Constitutional Order, Hong Kong 1997, S. 1 ff.; vgl. auch Lin, $R$. , Von der chinesischen Konzeption "Ein Land, zwei Systeme", VRÜ, Bd. 23 (1990), S. 56 ff. 
in Verträgen von 1842 und 1860 endgültig und dauerhaft an das Vereinigte Königreich abgetreten wurden. ${ }^{5}$ Demgegenüber pachtete das Vereinigte Königreich mit Vertrag vom 6. Juni 1898 ein weiteres und größeres Gebiet des chinesischen Festlandes, die "New Territories", wobei dieser Vertrag vorsah, daß die Pacht nach einem Zeitraum von 99 Jahren auslaufen sollte. ${ }^{6}$ Ohne daß hier Mutmaßungen über die Beweggründe angestellt werden sollen, beschloß das Vereinigte Königreich bereits zu Beginn der achtziger Jahre, mit Ablauf des Pachtvertrages über die "New Territories" auch die abgetretenen Gebiete Hong Kong Island und Kowloon zurückzugeben. Die Rückgabe der gesamten Kronkolonie Hong Kong einschließlich der "New Territories" wurde bereits 1984 durch die "Joint Declaration of the United Kingdom of Great Britain and Northern Ireland and the Government of the People's Republic of China on the Question of Hong Kong" ${ }^{7}$ festgelegt, wobei sich die Volksrepublik China im Gegenzug mit gleichem Vertragswerk verpflichtet hat, das Wirtschaftssystem und die Rechtsordnung von Hong Kong für einen weiteren Zeitraum von 50 Jahren unangetastet zu lassen. Ein wesentlicher Schritt bei der Vorbereitung zur Rückübertragung von Hong Kong erfolgte 1990 mit der Verabschiedung des "Basic Law of the Hong Kong Special Administrative Region of the People's Republic of China" ${ }^{8}$ durch den Nationalen Volkskongreß. Das "Basic Law", welches entsprechend der Verkündung durch den Präsidenten der Volksrepublik China mit dem Souveränitätswechsel am 1. Juli 1997 in Kraft getreten ist, stellt gleichsam die "Verfassung" für die Sonderverwaltungszone Hong

5

6

7

Nachfolgend: "Joint Declaration". Die "Joint Declaration" wurde am 26. September 1984 von Vertretern des Vereinigten Königreichs sowie der Volksrepublik China paraphiert, am 19. Dezember 1984 von Premierministerin Margaret Thatcher und Ministerpräsident Zhao Ziyang unterfertigt und schließlich am 27. Mai 1985 durch den Austausch der Ratifikationsurkunden wirksam. Mit der "Joint Declaration" haben die Regierungen des Vereinigten Königreichs und der Volksrepublik China Eckwerte für die grundlegende politische Richtung in der Sonderverwaltungszone Hong Kong festgeschrieben. Der Text ist abgedruckt bei Ress, G., The Legal Status of Hong Kong after 1997, ZaöRV 1986, S. 647 ff. (682 ff.); ferner Scheuer, M., Die Rechtslage von Hong Kong und Macao nach den "Gemeinsamen Erklärungen" vom 19. Dezember 1984 und 13. April 1987, Fn. 3, S. 231 ff.; eine deutsche Übersetzung findet sich im Europa-Archiv Folge 22/1984, S. $630 \mathrm{ff}$.

Nachfolgend: "Basic Law". Das "Basic Law" wurde in der dritten Sitzung des siebten Nationalen Volkskongresses der Volksrepublik China (nachfolgend: Nationaler Volkskongreß) am 4. April 1990 verabschiedet und am selben Tag vom Präsidenten der Volksrepublik China, Yang Shangkun, mit dem 1. Juli 1997 als Tag des Inkrafttretens per Dekret verkündet. Der Wortlaut ist abgedruckt bei International Legal Materials (ILM), Documents 1990 (Vol. XXIX), S. 1519 ff. mit Einführung $K u$, C., a.a.O., S. 1511 ff.; sowie Odrich, P., Basic Law of Hong Kong, JÖR, Bd. 39 (1990), S. 617 ff. Eine Bibliographie findet sich bei Wesley-Smith, P., The Basic Law - A Bibliography of the Hong Kong Transition, Hong Kong Law Journal, Vol. 27 (1997), Part 2, S. 248 ff. 
Kong dar. ${ }^{9}$ Weitere vorbereitende Maßnahmen zur Rückgabe von Hong Kong waren die Einrichtung der "Sino-British Joint Liaison Group", die Einigung über die frühzeitige Einrichtung des "Court of Final Appeal" sowie die Einberufung von "Preparatory Committee" und "Provisional Legislative Council". ${ }^{10}$ Die zuvor durchaus konstruktiven gemeinsamen Bemühungen der Volksrepublik China und des Vereinigten Königreichs verschlechterten sich, als der letzte Gouverneur von Hong Kong, Christopher Patten, bei den Wahlen zum Legislative Council im Jahr 1995 teilweise demokratische Strukturen einführte. ${ }^{11}$

Vor allem wenn man sich das große Interesse vor und auch während der Übergabe der ehemaligen Kronkolonie vergegenwärtigt, so ist es zwischenzeitlich ruhig geworden um die jetzige Sonderverwaltungszone Hong Kong. In der Tat ist der Wechsel selbst ohne spektakuläre Konflikte erfolgt. Der teilweise befürchtete "big bang" ist ebenso ausgeblieben wie eine radikale Veränderung der wirtschaftlichen Ausrichtung Hong Kongs durch die Zentralregierung in Peking. Von einem Zustand völliger Normalität läßt sich - zumal aus juristischer Sicht - jedoch nicht sprechen. ${ }^{12}$ Die Frage, wieviel Autonomie der Sonderverwaltungszone Hong Kong tatsächlich unter dem Prinzip "One Country Two Systems" ${ }^{13}$ zukommt, und die Art und Weise, wie die Behörden einerseits in der Volksrepublik China und andererseits in der Sonderverwaltungszone Hong Kong Konflikte bei den innerstaatlichen Kompetenzabgrenzungen bewältigen, ist so aktuell wie kaum zuvor. ${ }^{14}$

Eine Analyse der einzelnen Artikel befindet sich bei Scheuer, M., Die Rechtslage von Hong Kong und Macao nach den "Gemeinsamen Erklärungen" vom 19. Dezember 1984 und 13. April 1987, Fn. 3, S. $117 \mathrm{ff}$.

$\mathrm{Zu}$ diesen Gremien und deren Aufgaben vgl. im folgenden.

Ausführlich zu den rechtlichen und tatsächlichen Rahmenbedingungen des Souveränitätswechsels vgl. Yash Ghai, Hong Kong's New Constitutional Order, Fn. 4, S. 35 ff. und S. 473 ff.; Scheuer, $M$., Die Rechtslage von Hong Kong und Macao nach den "Gemeinsamen Erklärungen" vom 19. Dezember 1984 und 13. April 1987, Fn. 3, S. 48 ff.; Farny, T., Die Rückgabe Hong Kongs an die VR China, Wiesbaden 1997, S. 67 ff.; Luthra, T., Hong Kong: Von der britischen Kronkolonie zur Sonderverwaltungszone in der Volksrepublik China, RIW 1997, S. 625 ff.

Zur Unsicherheit bezüglich der rechtlichen Rahmenbedingungen in der Sonderverwaltungszone Hong Kong statt vieler Wild, D., Legal grey areas clouding contracts, South China Morning Post vom 6. Juli 1997.

Ausführlich zu dem zunächst mit Blick auf die Republik China (Taiwan) geprägten System "One Country Two Systems" Lin, R., Von der chinesischen Konzeption "Ein Land, zwei Systeme", VRÜ, Bd. 23 (1990), S. 56 ff.

14 Vgl. etwa die Untersuchung von Cheung, A., From Colony to Special Admistrative Region: Issues of Hong Kong's Autonomy within a Centralized Authoritarianistic State, VRÜ, Bd. 31 (1998), S. $302 \mathrm{ff}$. 
Um so mehr erstaunt es, daß das Urteil des "Court of Appeal of the High Court" ${ }^{15}$ in der Rechtssache der Sonderverwaltungszone Hong Kong gegen David Ma Wai Kwan und andere ${ }^{16}$, welches neben weiteren Fragen insbesondere die Fortgeltung des "Common Law" in der Sonderverwaltungszone Hong Kong zum Gegenstand hatte, im deutschsprachigen Raum auf nur geringes Interesse gestoßen ist.

Mit vorbezeichnetem Urteil nimmt die zur Entscheidung berufene Kammer nicht nur ausführlich Stellung zu den im Vorfeld der Rückgabe viel diskutierten Fragen der weiteren Geltung des kodifizierten Rechts und des "Common Law" in der Sonderverwaltungszone Hong Kong, sondern äußert sich darüber hinaus zu den Grenzen der Jurisdiktionsgewalt der lokalen Gerichte. Ferner behandelt das Urteil mit den Ausführungen zur Rechtmäßigkeit der "Provisional Legislature"17 und deren Gesetzgebungsakten grundlegende staatsrechtliche Fragen, die das Verhältnis der Volksrepublik China und der Sonderverwaltungszone Hong Kong betreffen. Die "Provisional Legislature" wurde nach dem Souveränitätswechsel Gesetzgebungsorgan in der Sonderverwaltungszone Hong Kong und bezeichnet sich seither als "Provisional Legislative Council". Bemerkenswert ist, daß die "Provisional Legislature" bereits am 24. März 1996 - und damit mehr als ein Jahr vor dem Souveränitätswechsel über Hong Kong - als vorläufiges Gesetzgebungsorgan für die Sonderverwaltungszone Hong Kong von dem "Preparatory Committee" 18 eingerichtet wurde und zunächst (in der Zeit vor dem Souveränitätswechsel) von Shenzhen - auf dem Gebiet der Volksrepublik China - aus Gesetzesentwürfe für die spätere Sonderverwaltungszone Hong Kong vorbe-

Nachfolgend: "Court of Appeal". Zur Bezeichnung der Gerichte in der Kronkolonie Hong Kong und in der Sonderverwaltungszone Hong Kong sowie zum Instanzenzug vgl. Law Society of Hong Kong, Conf usion Illusion, Hong Kong Lawyer, August 1997, S. 4.

Urteil vom 29. Juli 1997 = "Reservation of Question of Law No. 1 of 1997 between HKSAR (Applicant) and MA WAI-KWAN, David; CHAN KOK-WAI, Donny und TAM KIM-YUEN (Respondents)"; abgedruckt in: Gerard McCoy, QC (General Editor), Hong Kong Cases Part 4, 1997, S. 315 ff. (nachfolgend: [1997] 2 HKC S. [...]).

Zur Einrichtung der "Provisional Legislature" am 24. März 1996 durch das "Preparatory Committee" vgl. Yash Ghai, Hong Kong's New Constitutional Order, Fn. 4, S. 273 u. 275 ff.; ders., Dark day for our rights, South China Moming Post vom 30. Juli 1997; ferner Buddle, C., Averting legal vacuum main issue: judge, South China Moming Post vom 30. Juli 1997 und Pearsons, C., Court 'must accept' body, South China Moming Post vom 23. Juli 1997.

Das "Preparatory Committee", dessen zuletzt 150 Mitglieder teilweise aus Hong Kong und teilweise aus der Volksrepublik China stammten, wurde am Tag der Verabschiedung des "Basic Law" (vgl. Fn. 8) in der dritten Sitzung des siebten Nationalen Volkskongresses am 4. April 1990 eingerichtet. Das "Preparatory Committee" hatte zur Aufgabe, einen reibungslosen Übergang Hong Kongs von der britischen Kronkolonie zur Sonderverwaltungszone in der Volksrepublik China sicherzustellen und insbesondere Maßnahmen zur Errichtung der ersten Regierung und des ersten Gesetzgebungsorgans in der Sonderverwaltungszone zu treffen. Zum "Preparatory Committee" Yash Ghai, Hong Kong's New Constitutional Order, Fn. 4, S. 273 f. und S. 66; Buddle, C., Averting legal vacuum main issue: judge, South China Moming Post vom 23. Juli 1997. 
reitet hat. ${ }^{19}$ Nach dem Souveränitätswechsel trat die "Provisional Legislature" an die Stelle des "Legislative Council", das - seit 1995 teilweise demokratisch legitimiert - bis zum Souveränitätswechsel neben dem Gouverneur zuständiges Gesetzgebungsorgan in Hong Kong war. Ebenso traten die von der "Provisional Legislature" in der Zeit vor dem Souveränitätswechsel verabschiedeten Gesetzesentwürfe in Kraft. ${ }^{20}$

\section{Das Verfahren in der Rechtssache der Sonderverwaltungszone Hong Kong gegen David Ma und andere}

Das Ausgangsverfahren betraf ein strafgerichtliches Verfahren, welches bereits im August 1995 gegen die Beklagten vor dem "Court of First Instance" in Hong Kong eingeleitet wurde. Den Angeklagten wurde Prozeßbetrug vorgeworfen, ${ }^{21}$ wobei festzustellen bleibt, daß der Prozeßbetrug in Hong Kong nicht in einer ausdrücklichen Kodifikation ("statutory law") unter Strafe gestellt ist, sondern die Strafbarkeit sich aus dem "Common Law" ${ }^{\prime 2}$ ergibt. $^{23}$ In der Folge zog sich das Verfahren bis über den Zeitpunkt des Souveränitätswechsels hinaus und wurde dann vor dem "Court of First Instance" unter dem im wesentlichen gleichen Klagevorwurf weiterbetrieben. ${ }^{24}$ Die Verteidigung beantragte, unter Berücksichtigung der "Hong Kong Reunification Ordinance" ${ }^{25}$, des "Basic Law" ${ }^{26}$ und der

19

20

Vgl. im einzelnen Chen, A., The Provisional Legislative Council of the SAR, Hong Kong Law Journal, Vol. 27 (1997), Part 1, S 1 ff.

Zur Rechtmäßigkeit der "Provisional Legislature" und deren Gesetzgebungsakten vgl. unten V. 4. und Yash Ghai, Hong Kong's New Constitutional Order, Fn. 4, S. 275 ff., der darauf hinweist, daß das Verhalten des "National People's Congress of the People's Republic of China" und dessen Ständigem Ausschuß in diesem Zusammenhang wenig Beachtung des Basic Law erkennen lassen (a.a.O., S. 280); ferner Tsui, C. / Won, L., 'Darkest hour' for democracy, South China Morning Post vom 22. Dezember 1996; Cheung, S., Shadow body illegal says absentee voter, South China Moming Post vom 22. Dezember 1996; Yeung, C., Patten calls vote by 400 'bizarre farce', South China Moming Post vom 22. Dezember 1996; Choy, L., HK backs shadow body, says Tung, South China Moming Post vom 22. Dezember 1996.

Der Anklagevorwurf lautete "conspiracy to pervert the course of public justice, contrary to common law", Vgl. [1997] 2 HKC S. 315 (320 f.).

Vereinfacht dargestellt handelt es sich bei dem "Common Law" um eine Fülle von nicht kodifizierten Rechtssätzen, die sich im wesentlichen aus der Urteilspraxis der Gerichte in den "Common Law-Staaten" ergeben. Vgl. mit zahlreichen weiteren Nachweisen Lundmark, Th., Juristische Technik und Methodik des Common Law, Münster 1998.

Vgl. [1997] 2 HKC S. 315 (320 f.).

Die Anklage gegen den dritten Angeklagten wurde in einem weiteren Anklagepunkt vorläufig eingestellt, ohne daß es für die Ausführungen in diesem Beitrag hierauf ankäme.

"Hong Kong Special Administrative Region Ordinance No. 110 of 1997", welche auf den in der 24. Sitzung des Ständigen Ausschusses des achten Nationalen Volkskongresses vom 23. Februar 1997 gefaßten Beschluß zurückgeht. Abgedruckt bei Yash Ghai, Hong Kong's New Constitutional Order, Fn. 4, S. 99 ff. 
Unanwendbarkeit des "Common Law" die Angeklagten freizusprechen. In der Hauptsache berief sich die Verteidigung darauf, daß die Grundlage der Strafbarkeit, nämlich das "Common Law", mit dem Souveränitätswechsel weggefallen sei, da der notwendige (ausdrückliche) Akt einer "Adoption" in bezug auf die Sonderverwaltungszone Hong Kong nicht rechtswirksam vorgenommen worden sei. Die Vertreter der Anklage waren demgegenüber der Ansicht, daß das "Common Law" ohne weiteres, das heißt ohne ausdrücklichen Adoptionsakt, Teil der Rechtsordnung in der Sonderverwaltungszone Hong Kong geworden sei. Entsprechend dem lokalen Verfahrensrecht ${ }^{27}$ wurde auf Antrag der Anklagevertreter die Frage der (weiteren) Gültigkeit des "Common Law" in der Sonderverwaltungszone Hong Kong dem "Court of Appeal" zur Vorabentscheidung vorgelegt. ${ }^{28}$

\section{Die Argumentation der Verteidigung und der Vertretung der Anklage}

\section{Die Argumentation der Verteidigung}

Zur Begründung, das "Common Law" sei nicht Teil der Rechtsordnung in der Sonderverwaltungszone Hong Kong, beruft sich die Verteidigung auf drei wesentliche Argumente. ${ }^{29}$ Zum einen sei in Art. 160 des "Basic Law" vorgesehen, daß das vor dem Souveränitätswechsel in Hong Kong gültige Recht, zu dem auch das "Common Law" zählt, durch einen ausdrücklichen Übertragungsakt "adoptiert" werden müsse. ${ }^{30}$ Die Formulierung in Art. 160 des "Basic Law" "[...] shall be adopted [...]" verdeutliche, daß ein ausdrücklicher Rechtsakt vorausgesetzt werde, ohne den das zuvor in Hong Kong gültige Recht nicht Teil der Rechtsordnung in der Sonderverwaltungszone Hong Kong werden könne. ${ }^{31}$ Ein solcher "Übertragungsakt" sei nicht in wirksamer Weise vorgenommen worden.

26

27

28

29

30

31

Vgl. die Nachweise oben Fn. 8.

Section 81 der "Criminal Procedure Ordinance", Cap. 221.

Nachdem die Vertreter der Anklage dem "Court of Appeal" zunächst fünf Fragen zur Vorabentscheidung vorgelegt hatten, beschränkten sie sich später auf zwei Fragen: 1. Ob die Strafbarkeit eines Prozeßbetruges auf der Grundlage des "Common Law" Teil der Rechtsordnung in der Sonderverwaltungszone Hong Kong sei, und 2. ob die Angeklagten überhaupt gezwungen seien, auf die Anklage einzugehen und ein Verfahren gegen sich gelten zu lassen.

gl. [1997] 2 HKC S. 315 (320 f.).

Art. 160 des "Basic Law" lautet (Hervorhebung durch den Autor) "Upon the establishment of the Hong Kong Special Administrative Region, the laws previously in force in Hong Kong shall be adopted as laws of the Region except those which the Standing Committee of the National People's Congress declares to be in contravention of this Law. If any laws are later discovered to be in contravention of this Law, they shall be amended or cease to have force in accordance with the procedure as prescribed by this Law." Vgl. [1997] 2 HKC S. 315 (322 und 326).

Das Verlangen nach einem solchen "ausdrücklichen Übertragungsakt" läßt sich vergleichen mit der von den Befürwortern der dualistischen Lehre für erforderlich befundenen Transformation des 
Wie die Verteidigung weiter ausführt, könne die Entscheidung des Ständigen Ausschusses des Nationalen Volkskongresses vom 23. Februar $1997^{32}$ keinen wirksamen Übertragungsakt darstellen. Zwar werde in dieser Entscheidung des Ständigen Ausschusses ausdrücklich die Weitergeltung des "Common Law" in der Sonderverwaltungszone Hong Kong festgeschrieben. Zugleich werde in den drei Annexen zu der genannten Entscheidung eine detaillierte Regelung darüber getroffen, welche der vor dem Souveränitätswechsel in Hong Kong gültigen Kodifikationen nicht Teil der Rechtsordnung in der Sonderverwaltungszone Hong Kong werden sollen. ${ }^{33} \mathrm{Zu}$ den nach Annex 1 nicht anwendbaren Rechtsnormen gehört insbesondere die "Application of English Law Ordinance" ${ }^{34}$, die nach Auffassung der Verteidigung Grundlage für die Geltung des "Common Law" in Hong Kong vor dem Souveränitätswechsel war. Durch die Zurückweisung der "Application of English Law Ordinance" habe der Ständige Ausschuß zu erkennen gegeben, daß das englische Rechtssystem (u. a. das "Common Law") nicht Rechtsgrundlage in der Sonderverwaltungszione Hong Kong sein solle.

Ferner stelle auch die "Hong Kong Reunification Ordinance" ${ }^{35}$, die auf die Entscheidung des Ständigen Ausschusses des Nationalen Volkskongresses vom 23. Februar 1997 Bezug nimmt und darüber hinaus in Sektion 5 und Sektion $7^{36}$ grundsätzlich die Weitergeltung

Völkerrechts in innerstaatliches Recht. Dabei wird nicht übersehen, daß es sich bei dem "Common Law" nicht um Rechtssätze des Völkerrechts handelt. Die rechtliche Situation bei der Übernahme des vor dem Souveränitätswechsel in Hong Kong gültigen Rechts in die Sonderverwaltungszone läßt sich auch nicht vollständig mit der Situation bei der Übernahme von Völkerrechtsregeln in eine innerstaatliche Rechtsordnung vergleichen (hierzu ausführlich unten V. 2.). Zur Transformation vgl. Bleckmann, A., Grundgesetz und Völkerrecht, Berlin 1975, S. 217 ff. und 264 ff.; Geiger, R., Grundgesetz und Völkerrecht, 2. Aufl., München 1994, S. 15 ff. und 158 ff.; Seidl-Hohenveldern, I., Völkerrecht, 9. Aufl., Köln / Berlin / Bonn / München, 1997, RdNr. 556 ff.; Kunig, P. in: Graf Vitztum (Hrsg.) Völkerrecht, Berlin / New York 1997, II, 28 ff.

"Decision of the Standing Committee of the National People's Congress on Treatment of the Laws Previously in Force in Hong Kong in Accordance with Article 160 of the Basic Law of the Hong Kong Special Administrative Region of the People's Republic of China", welche von dem Ständigen Ausschuß des achten Nationalen Volkskongresses auf dessen 24. Sitzung verabschiedet wurde. Eine englische Übersetzung findet sich bei Yash Ghai, Hong Kong's New Constitutional Order, Fn. 4, S. 499 ff.

Vgl. [1997] 2 HKC S. 315 (322 und 328).

"Application of English Law Ordinance", Cap. 88 von 1966. Genauer hierzu unten IV.

Hong Kong Special Administrative Region Ordinance No. 110 of 1997.

Durch Sektion 5 der "Hong Kong Reunification Ordinance" wird an die Begriffsbestimmungen in Sektion 2 eine weitere Sektion 2A. mit folgendem Wortlaut angefügt: "'2A. Laws previously in force (1) All laws previously in force shall be construed with such modifications, adaptions, limitations and exceptions as may be necessary so as not to contravene the Basic Law and to bring them in conformity with the status of Hong Kong as a Special Administrative Region of the People's Republic of China. (2) Without prejudice to the generality of subsection (1), in any Ordinance - [...] (e) provisions applying English law may continue to be applicable by reference thereto as a transitional arrangement pending their amendment by the Hong Kong Special 
des vor dem Souveränitätswechsel in Hong Kong gültigen Rechts (und damit auch des "Common Law") in der Sonderverwaltungszone vorsieht, keinen wirksamen Übertragungsakt dar. Die "Hong Kong Reunification Ordinance" sei von dem "Provisional Legislative Council" ${ }^{37}$ erlassen worden, obwohl das "Provisional Legislative Council" unter Verstoß gegen das "Basic Law" zustande gekommen und damit nicht für die Gesetzgebung in der Sonderverwaltungszone Hong Kong zuständig sei. ${ }^{38}$ Aus der Entscheidung des Ständigen Ausschusses vom 23. Februar 1997 und der "Hong Kong Reunification Ordinance", in denen - wenn auch in unwirksamer Weise - ausdrückliche Regelungen zur Weitergeltung des "Common Law" getroffen worden seien, sei ein eindeutiger Hinweis darauf zu entnehmen, daß auch der Nationale Volkskongreß von der Notwendigkeit eines "Adoptionsaktes" ausging, ohne den das vor dem Souveränitätswechsel in Hong Kong gültige Recht (mithin auch das "Common Law") nicht Teil der Rechtsordnung in der Sonderverwaltungszone Hong Kong werden könne.

\section{Die Argumentation der Anklage}

Demgegenüber argumentiert die Anklage, daß das "Basic Law" selbst und unmittelbar die Weitergeltung des "Common Law" als Teil der Rechtsordnung in der Sonderverwaltungszone Hong Kong vorsehe, ohne daß es eines weiteren Aktes der "Adoption" bedurft hätte. ${ }^{39}$ Eine ausdrückliche Entscheidung sei auf der Grundlage von Art. 160 des "Basic Law" nur erforderlich hinsichtlich der gesetzlichen Bestimmungen, die vor dem Souveränitätswechsel Teil der Rechtsordnung von Hong Kong waren, aber nicht Teil der Rechtsordnung in der Sonderverwaltungszone Hong Kong werden sollten. Alleine diese Bestimmung der in der Sonderverwaltungszone Hong Kong unanwendbaren Vorschriften habe der Ständige Ausschuß des Nationalen Volkskongresses in seiner Entscheidung vom 23. Februar 1997 getroffen. ${ }^{40}$ Auf die unmittelbar aus Art. 160 des "Basic Law" folgende Gültigkeit des

Administrative Region through the Legislature thereof, provided that they are not prejudicial to the sovereignty of the People's Republic of China and do not contravene the provisions of the Basic Law. [...] (4) In this section - 'Basic Law' means the Basic law of the Hong Kong Special Administrative Region of the People's Republic of China; 'laws previously in force' means the common law, rules of equity, Ordinances, subsidiary legislation and customary law in force immediately before 1 July 1997 and adopted as laws of the Hong Kong Special Administrative Region.'." Sektion 7 der Hong Kong Reunification Ordinance lautet: "Maintenance of previous laws (1) The laws previously in force in Hong Kong, that is the common law, rules of equity, Ordinances, subsidiary legislation and customary law, which have been adopted as the laws of the HKSAR, shall continue to apply."

Zum "Provisional Legislative Council" vgl. oben Fn. 17 und Fn. 20.

Vgl. [1997] 2 HKC S. 315 (336 f.).

Vgl. [1997] 2 HKC S. 315 (322 u. 327).

Vgl. [1997] 2 HKC S. 315 (322 f.). 
"Common Law" als Teil der Rechtsordnung in der Sonderverwaltungszone Hong Kong habe indessen weder die Entscheidung des Ständigen Ausschusses noch deren Bestätigung durch die "Hong Kong Reunification Ordinance" eine Auswirkung. Daher komme es auch nicht auf die Frage an, ob die Einrichtung des "Provisional Legislative Council" in rechtmäßiger Weise erfolgte. Jedenfalls falle die Entscheidung über die Rechtmäßigkeit des "Provisional Legislative Council" nicht in die Jurisdiktionskompetenz der Gerichte in der Sonderverwaltungszone Hong Kong. ${ }^{41}$ Die Einrichtung des "Provisional Legislative Council" durch das "Preparatory Committee" stelle einen Rechtsakt dar, den die zuständigen Organe der Volksrepublik China als Souverän getroffen hätten. Ein solcher Rechtsakt des Souveräns unterliege nicht der Rechtsprechungsgewalt regionaler Gerichte, wie etwa der Gerichte in der Sonderverwaltungszone Hong Kong. ${ }^{42}$

\section{Das Urteil des "Court of Appeal"}

Die zur Entscheidung berufene Kammer des "Court of Appeal" folgt in ihrem Urteil im wesentlichen der Argumentation der Anklage und stellt fest, daß das "Common Law" auch nach dem Souveränitätswechsel Teil der Rechtsordnung in der Sonderverwaltungszone Hong Kong ist. In seiner Urteilsbegründung führt der vorsitzende Richter der Kammer ${ }^{43}$ unter Berufung auf den englischen und chinesischen Wortlaut, ${ }^{44}$ den Normkontext, die Intention sowie die Entstehungsgeschichte ${ }^{45}$ zu Art. 160 des "Basic Law" aus, daß diese Norm selbst und unmittelbar die Weitergeltung des "Common Law" in der Sonderverwaltungszone Hong Kong sicherstelle, ohne daß es eines weiteren Rechtsaktes bedürfe. ${ }^{46}$ Dem stehe auch nicht die Entscheidung des Ständigen Ausschusses des Nationalen Volkskongresses vom 23. Februar 1997 entgegen, deren Zweck die Festlegung derjenigen Rechtssätze sei, die nicht Teil der Rechtsordnung in der Sonderverwaltungszone Hong Kong

41

42

43

44

45

46

Vgl. [1997] 2 HKC S. 315 (333 ff.). Mortimer als Beisitzenden. Hong Kong, VRÜ, Bd. 26 (1993), 275 ff.

Die Kammer beim "Court of Appeal" bestand in diesem Verfahren aus drei Richtern, mit dem "Chief Judge" Patrick Chan Siu-oi als Vorsitzendem und den Richtern Gerald Nazareth und Barry

4 Da das "Basic Law" als Gesetzgebungsakt der Volksrepublik China in chinesischer Sprache verabschiedet wurde, ist der chinesische Gesetzestext (und nicht die englische Übersetzung) maßgebliche Quelle für die (Wortlaut-) Auslegung der einzelnen Bestimmungen. Zum Sprachproblem allgemein Pasternak, V., Chinesisch als Rechtssprache im Kontext des Common Law: Der Fall

Unter Berücksichtigung der "Joint Declaration".

Vgl. [1997] 2 HKC S. 315 (326 ff. u. 330). 
werden sollen. Die gleichzeitig getroffene ausdrückliche Bestätigung, daß unter anderem das "Common Law" weiterhin gelten soll, sei rein deklaratorisch. ${ }^{47}$

Dem stimmen auch die beisitzenden Richter zu, ${ }^{48}$ wobei sie ergänzend darauf hinweisen, daß die Entscheidung des Ständigen Ausschusses des Nationalen Volkskongresses vom 23. Februar 1997 ebenso wie die Verabschiedung der "Hong Kong Reunification Ordinance" sieben Jahre nach der Verabschiedung des "Basic Law" erfolgt seien. Die späteren Entscheidungen des Nationalen Volkskongresses ließen sich jedoch, ebenso wie nachfolgende gesetzliche Regelungen, nur in sehr beschränktem Umfang bei der Interpretation des "Basic Law" heranziehen. ${ }^{49}$ Ebenso habe die Zurückweisung der "Application of English Law Ordinance" keine Auswirkung auf die Anwendbarkeit des "Common Law", da dieses bereits vor dem Inkrafttreten der "Application of English Law Ordinance" Teil der Rechtsordnung in Hong Kong gewesen sei und insoweit eine weitergehende Anwendungslegitimation habe. 50

Da die Kammer zu der Auffassung gelangt ist, das "Common Law" gelte unmittelbar auf der Grundlage von Art. 160 des "Basic Law", erfolgen die weiteren Ausführungen in der Urteilsbegründung zur "Hong Kong Reunification Ordinance", zum "Provisional Legislative Council" und zur Jurisdiktionsgewalt der Gerichte in der Sonderverwaltungszone Hong Kong als "obiter dicta". 51 Für den Fall, daß das "Basic Law" nicht die "automatische" Weitergeltung des "Common Law" in der Sonderverwaltungszone Hong Kong vorsehe, stellt das Gericht ausdrücklich fest, daß das "Common Law", jedenfalls auf der Grundlage der "Hong Kong Reunification Ordinance" 52 , Teil der Rechtsordnung in der Sonderverwaltungszone Hong Kong geworden sei. Die Kammer begründet diese Ansicht mit der Feststellung, daß die Einrichtung des "Provisional Legislative Council" durch das "Preparatory Committee" und den Nationalen Volkskongreß ein Rechtsakt der zuständigen

Vgl. [1997] 2 HKC S. 315 (328). So auch Yash Ghai, The Continuity of Laws and Legal Rights and Obligations in the SAR, Hong Kong Law Joumal, Vol. 27 (1997), Part 2, S. 136 ff. (141), der darauf hinweist, daß solche deklaratorischen Wiederholungen in der chinesischen Legislativpraxis nicht ungewöhnlich sind. Fermer Wacks, R., The Future of the Common Law, Hong Kong Law Journal, Vol. 27 (1997), Part 2, S. 129 f.

Gemäß dem in der Sonderverwaltungszone Hong Kong gültigen Verfahrensrecht gibt jedes Mitglied der Kammer eine eigene Urteilsbegründung ab.

49

50

Vgl. [1997] 2 HKC S. 315 (346).

51

Vgl. [1997] 2 HKC S. 315 (328 f.).

Das geht auch ausdrücklich aus dem Urteil hervor, in welchem die Ausführungen zur Jurisdiktionsgewalt der Gerichte in der Sonderverwaltungszone Hong Kong und zur Rechtmäßigkeit des "Provisional Legislative Council" als "obiter" gekennzeichnet sind. Vgl. [1997] 2 HKC S. 315 (318 f.).

52

Im einzelnen beruft sich die Kammer auf den Titel sowie Sektionen 5, 7, 8, 10 und 15 der "Hong Kong Reunification Ordinance". 
Organe in der Volksrepublik China als Souverän über die Sonderverwaltungszone Hong Kong sei, der durch die Gerichte in der Sonderverwaltungszone Hong Kong nur in beschränktem Umfang nachprüfbar wäre. ${ }^{53}$

Die Kammer betont, daß regionale Gerichte unzuständig seien, wenn es darum gehe, diè Gültigkeit von Rechtsakten, die ihren Ursprung beim Souverän finden, auf ihre Rechtmäßigkeit zu überprüfen. Ebensowenig stünde den regionalen Gerichten zu, die Motive des Souveräns zu hinterfragen. Mithin sei es den Gerichten in der Sonderverwaltungszone Hong Kong verwehrt, die Einrichtung des "Provisional Legislative Council" durch das "Preparatory Committee" auf die Vereinbarkeit mit dem "Basic Law" zu überprüfen. Allerdings verbleibe den Gerichten in der Sonderverwaltungszone Hong Kong die Kompetenz nachzuprüfen, ob es überhaupt einen Rechtsakt des Nationalen Volkskongresses bezüglich der Einrichtung des "Peparatory Committee" gegeben habe, ob ferner eine Entscheidung des "Preparatory Committee" zur Einrichtung des "Provisional Legislative Council" vorgelegen habe und schließlich, ob das "Provisional Legislative Council" tatsächlich das Organ sei, welches infolge der Entscheidungen des Nationalen Volkskongresses und des "Preparatory Committee" errichtet worden ist. ${ }^{54}$

In der weiteren Urteilsbegründung geht die Kammer vom Vorliegen der genannten Voraussetzungen aus und be jaht somit im Rahmen der selbstgesteckten Grenzen der Jurisdiktionsgewalt die ordnungsgemäße Einrichtung des "Provisional Legislative Council". Insbesondere hebt die Kammer hervor, daß die Volksrepublik China, deren höchstes Staatsorgan der Nationale Volkskongreß ist, ${ }^{55}$ der Souverän über die Sonderverwaltungszone Hong Kong sei. Die Einrichtung und Mandatierung des "Preparatory Committee" gehe unmittelbar auf den Nationalen Volkskongreß und dessen Ständigem Ausschuß zurück. Ferner sei das "Preparatory Committee" beauftragt worden, die Errichtung der Sonderverwaltungszone Hong Kong vorzubereiten. Hierzu gehöre auch die Einrichtung des "Provisional Legislative Council" als Gesetzgebungsorgan in der Sonderverwaltungszone Hong Kong. ${ }^{56}$

53

54

55

56

Vgl. [1997] 2 HKC S. 315 (330 ff.).

Vgl. [1997] 2 HKC S. 315 (334 f.).

Vgl. Art. 57 und Art. 59 der Verfassung der Volksrepublik China in der Fassung vom 4. Dezember 1982, in englischer Sprache abgedruckt bei Foreign Language Press Beijing, The Constituition of the People's Republic of China, Beijing 1983. Eine deutsche Übersetzung der Verfassung der Volksrepublik China findet sich bei Scheuer, M., Die Rechtslage von Hong Kong und Macao nach den "Gemeinsamen Erklärungen" vom 19. Dezember 1984 und 13. April 1987, Fn. 3, S. 209 ff. Zu der Bedeutung des Nationalen Volkskongresses und dessen Ständigem Ausschuß auf der Grundlage der Verfassung der Volksrepublik China vgl. Yash Ghai, Hong Kong's New Constitutional Order, Fn. 4, S. $101 \mathrm{ff}$.

Vgl. [1997] 2 HKC S. 315 (340 ff.). 
Die Kammer hebt hervor, daß es sich bei dem "Provisional Legislative Council" nicht um das erste Gesetzgebungsorgan in der Sonderverwaltungszone Hong Kong handele, wie es Art. 68 des "Basic Law" und dessen Annex II vorsehen. Vielmehr unterstütze das "Provisional Legislative Council" die erste Regierung der Sonderverwaltungszone Hong Kong, die Regelungen des "Basic Law" und die Entscheidungen des Nationalen Volkskongresses zu implementieren. ${ }^{57}$ Die mit dem Souveränitätswechsel gleichzeitige Einrichtung des ersten Gesetzgebungsorgans gemäß Art. 68 des "Basic Law" und dessen Annex II sei nicht möglich gewesen, da die schlichte Übernahme des "Legislative Council" der Kronkolonie Hong Kong seit der teilweise demokratischen Wahl von 1995 für die Volksrepublik China nicht hinnehmbar gewesen sei. ${ }^{58}$ Ebenso hätten die zuständigen Behörden der Volksrepublik China, mithin das "Preparatory Committee", keine Möglichkeit gehabt, vor dem Souveränitätswechsel Wahlen zum ersten "Legislative Council" in der Sonderverwaltungszone Hong Kong vorzubereiten. ${ }^{59}$ Daher sei es gerade Aufgabe des "Provisional Legislative Council" - in Zusammenarbeit mit der Regierung - in der Sonderverwaltungszone Hong Kong bis spätestens 30. Juni 1998 die Voraussetzungen für die Wahl des ersten Gesetzgebungsorgans zu schaffen, welches im Einklang mit Art. 68 des "Basic Law" und Annex II zum "Basic Law" stehe. Die Einrichtung des "Provisional Legislative Council" sei ein "interim measure", wobei der Souverän die Kompetenz habe, eine solche "Zwischenmaßnahme" zu treffen. Insofern sei das "Provisional Legislative Council" tatsächlich und im Rahmen seiner Zuständigkeiten vom Nationalen Volkskongreß und dem "Preparatory Committee" errichtet worden. Darüber hinaus fehle es den Gerichten in der Sonderverwaltungszone Hong Kong an der Kompetenz, die Rechtmäßigkeit des "Provisional Legislative Council" zu überprüfen.

57

58

59

Vgl. [1997] 2 HKC S. 315 (341 f.).

Der Ständige Ausschuß des Nationalen Volkskongresses hat in seiner Entscheidung vom 31. August 1994 festgestellt, daß die Wahlvorbereitungen für das "Legislative Council" im Jahr 1994 gegen die "Joint Declaration", gegen das "Basic Law" und gegen Entscheidungen des Ständigen Ausschusses des Nationalen Volkskongresses verstießen. Gleichzeitig wurde klar, daß es keinen "through train" für das erste Gesetzgebungsorgan in der Sonderverwaltungszone Hong Kong geben würde. Unter dem Begriff des "through train" wurde die Möglichkeit verstanden, daß das letzte Legislativorgan der Kronkolonie Hong Kong, also das "Legislativ Council" (ohne Berücksichtigung der Kompetenzen des Gouverneurs), ohne sachliche oder personelle Veränderung auch das erste Legislativorgan in der Sonderverwaltungszone Hong Kong würde. Vgl. im einzelnen Chen, A., The Provisional Legislative Council of the SAR, Hong Kong Law Joumal, Vol. 27 (1997), Part 1, S. $1 \mathrm{ff}$.

Vgl. [1997] 2 HKC S. 315 (341 f.). 


\section{Kritische Anmerkungen zum Urteil des "Court of Appeal" \\ 1. Überblick}

Das Urteil des "Court of Appeal" in der Rechtssache der Sonderverwaltungszone Hong Kong gegen David Ma und andere verdient Zustimmung, insoweit es die Weitergeltung des "Common Law" in der Sonderverwaltungszone Hong Kong unmittelbar aufgrund des "Basic Law" bestätigt und die Notwendigkeit eines weiteren und ausdrücklichen Adoptionsaktes verneint. Bedauerlicherweise beläßt es das Gericht nicht bei dieser Feststellung, die für sich genommen vollkommen ausgereicht hätte, die vom "Court of First Instance" zur Entscheidung vorgelegten Fragen umfassend zu beantworten. ${ }^{60}$ Kritisch betrachtet werden müssen die als "obiter dicta" zu verstehenden weiteren Äußerungen des "Court of Appeal" zur rechtmäßigen Einrichtung des "Provisional Legislative Council" und insbesondere zur Beschränkung der Jurisdiktionsgewalt der Gerichte in der Sonderverwaltungszone Hong Kong. Die Feststellungen des "Court of Appeal" zu den beiden zuletzt genannten Aspekten sind, wie darzustellen sein wird, nicht nur in rechtlicher Hinsicht sehr zweifelhaft, sondern bedeuten auch in tatsächlicher Hinsicht und unter dem Grundsatz "One Country Two Systems" ein Indiz für das in hohem Maß eingeschränkte Autonomieverständnis der Gerichte in der Sonderverwaltungszone Hong Kong. ${ }^{61}$

\section{Anwendung des "Common Law" unmittelbar auf der Grundlage des "Basic Law"}

Zuzustimmen ist dem "Court of Appeal", daß das "Common Law" unmittelbar aufgrund von Art. 160 des "Basic Law" Teil der Rechtsordnung in der Sonderverwaltungszone Hong Kong geworden ist, ohne daß es eines weiteren Rechtsaktes bedurft hätte. Dabei ist unerheblich, ob man den durch Art. 160 des "Basic Law" vorgenommenen Übertragungsakt als "Adoption", "Transformation" oder - wegen des eingeschränkten völkerrechtlichen Bezu-

60

So auch Yash Ghai, Dark day for our rights, South China Moming Post vom 30. Juli 1997, der ausführt: "These findings were sufficient to dispose of the case. Nevertheless the court, contrary to normal constitutional practice, proceeded to decide other constitutional questions."

61

So auch Cheung, A., From Colony to Special Admistrative Region: Issues of Hong Kong's Autonomy within a Centralized Authoritarianistic State, VRÜ, Bd. 31 (1998), S. 307; Yash Ghai, Dark day for our rights, South China Moming Post vom 30. Juli 1997; Choy, L. / Ku, G., Fears as 'bulwark of Basic Law falls', South China Moming Post vom 30. Juli 1997; Choy, L., Selfimposed limits could sacrifice autonomy, law academic warns, South China Moming Post vom 30. Juli 1997; dies., Political Judgements 'harm courts', South China Moming Post vom 30. Juli 1997; Ku, G., Fight goes on, says United Front, South China Moming Post vom 30. Juli 1997; Political Desk, Territory 'digging its own grave', South China Moming Post vom 31. Juli 1997; Ng, M., Decision that resonates, South China Morning Post vom 8. Aug. 1997; Li, A., Bar Chief hits at 'backward-looking' example in provisional legislature case, South China Moming Post vom 13. September 1997. 
ges - als einen Übertragungsakt eigener Art qualifiziert. ${ }^{62}$ Entscheidend ist vielmehr, daß Art. 160 des "Basic Law" die Weitergeltung unter anderem des "Common Law" in der Sonderverwaltungszone Hong Kong festlegt. Dabei bedurfte es unter Berücksichtigung des Wortlautes dieser Norm, insbesondere des Wortlautes in der maßgeblichen chinesischen Fassung, der Entstehungsgeschichte unter Berücksichtigung der "Joint Declaration" und mit Blick auf die Zielsetzung, die der Nationale Volkskongreß bei der Verabschiedung des "Basic Law" verfolgte, keines weiteren Übertragungsaktes. ${ }^{63}$ Zur weiteren Begründung dieser Ansicht wird auf die bereits skizzierte Argumentation der Anklagevertreter sowie auf die ebenfalls dargelegte ausführliche Urteilsbegründung der entscheidenden Kammer des "Court of Appeal" verwiesen.

\section{Die Jurisdiktionsgewalt der Gerichte in der Sonderverwaltungszone Hong Kong}

\section{a) Vorbemerkung}

Eine Beschränkung der Jurisdiktionsgewalt der Gerichte in der Sonderverwaltungszone Hong Kong - wie sie die zur Entscheidung berufene Kammer des "Court of Appeal" mit dem gegenständlichen Urteil vornimmt ${ }^{64}$ - ist im Ergebnis abzulehnen. Den Gerichten in der Sonderverwaltungszone Hong Kong muß die Rechtsprechungskompetenz zukommen, zumindest soweit es um die Überprüfung der "Basic Law"-Konformität solcher Rechtsakte geht, die ausschließlich Wirkung auf dem Gebiet der Sonderverwaltungszone Hong Kong entfalten. Eine andere Sichtweise bedeutet eine fehlerhafte Bewertung des "Basic Law" als Rechtsnorm mit Verfassungscharakter, die Aushöhlung der - unter der "Joint Declaration" und dem "Basic Law" postulierten - weitgehenden Autonomie der Sonderverwaltungszone Hong Kong von der Volksrepublik China und im Ergebnis die Verneinung eines Rechts-

Zur Abgrenzung der "Adoption" und "Transformation" vgl. Seidl-Hohenveldern, I., Völkerrecht, Fn. 31, RdNr. 539 ff.; Kunig, P., in Graf Vitztum (Hrsg.) Völkerrecht, Fn. 31, II, 28 ff.

63 Im einzelnen hierzu Yash Ghai, Hong Kong's New Constitutional Order, Fn. 4, S. 356 ff.; und ders., The Continuity of Laws and Legal Rights and Obligations in the SAR, Hong Kong Law Joumal, Vol. 27 (1997), Part 2, S. 136 ff. (139), der auf Art. 8 und Art. 18 des "Basic Law" abstellt und Art. 160 des "Basic Law" vor dem Hintergrund der "Joint Declaration" dahingehend interpretiert, daß der Nationale Volkskongreß nur berechtigt sei, bei der Gründung der Sonderverwaltungszone Hong Kong solche Kodifikationen zu benennen, die nicht Teil der Rechtsordnung in der Sonderverwaltungszone Hong Kong werden sollen. Umgekehrt gebe Art. 160 des "Basic Law" dem Nationalen Volkskongreß jedoch gerade keine Kompetenz, Feststellungen in positiver Hinsicht zu treffen. Femer Wacks, R., The future of the Common Law, Hong Kong Law Joumal, Vol. 27 (1997), Part 2, S. 129 ff.

Vgl. [1997] 2 HKC S. 315 (330 ff.) und oben IV. 
weges zur Überprüfung der Vereinbarkeit Hong Kong bezogener Rechtsakte des Nationalen Volkskongresses am Maßstab des "Basic Law". 65

Die Reichweite der Jurisdiktionsgewalt von Gerichten in der Sonderverwaltungszone Hong Kong ergibt sich aus der verfassungsrechtlichen Bedeutung des "Basic Law" und dem Verständnis der Autonomie im Sinne des "Basic Law" und der "Joint Declaration"66 . Die genannten Kodifikationen stellen neben allgemeinen völkerrechtlichen Grundsätzen die Grundlage für die Beurteilung des staatsrechtlichen Status der Sonderverwaltungszone Hong Kong und zugleich für das staatsrechtliche Verhältnis der Sonderverwaltungszone Hong Kong zur Volksrepublik China dar. Der von Deng Xiao Ping aufgestellte Grundsatz "One Country Two Systems" ist dabei eine Interpretationshilfe.

\section{b) Die "Joint Declaration"}

Bereits die "Joint Declaration" schreibt die weitgehende Autonomie der Sonderverwaltungszone Hong Kong von der Zentralregierung der Volksrepublik China ausdrücklich fest. Die "Joint Declaration" wurde im Dezember 1984 von der Premierministerin des Vereinigten Königreichs und dem Ministerpräsidenten der Volksrepublik China unterzeichnet. Als gemeinsame Erklärung des Vereinigten Königreichs und der Volksrepublik China stellt die "Joint Declaration" zusammen mit ihren Annexen ${ }^{68}$ einen völkerrechtlich verbindlichen Vertrag dar, der zum einen mit Ablauf des 30. Juni 1997 die Rückgabe der Kronkolonie Hong Kong und Kowloon sowie der "New Territories" vorsieht und zum anderen die Eck-

Hierzu sogleich und anschaulich Yash Ghai, Dark day for our rights, South China Morning Post vom 30. Juli 1997, der ausführt: "The decision opens the floodgates to any evasion, distortion or disregard of the Basic Law. [...] Hong Kong is no different from Shanghai or Fujian. Few would have expected such a rapid demise of 'one country two systems'."; sowie $N g, M$., Decision that resonates, South China Morning Post vom 8. August 1997, die ausführt: "In this context it is difficult to understand precisely what the Court of Appeal is referring to as an unchallengable act of the sovereign. If by these the Court of Appeal means that the NPC reserves an unlimited power to legislate for Hong Kong which it can exercise in any way and at any time it sees fit, without regard to anything provided in the Basic Law, then it would be better if the court made it clear while showing clearly the legal principles under the common law or Chinese Law which lead to this conclusion."; und Choy, L., Self-imposed limits could sacrifice autonomy, law academic warns, South China Morning Post vom 30. Juli 1997. Vgl. die Nachweise in Fn. 7.

Insgesamt drei Annexe: I. "Elaboration by the Government of the People's Republic of China of its Basic Policies regarding Hong Kong" (Erklärung der Regierung der Volksrepublik China zur grundlegenden politischen Richtung in bezug auf Hong Kong), II. "Sino-British Joint Liaison Group" (Gemeinsamer britisch-chinesischer Verbindungsausschuß) und III. "Land Leases" (grundstücksbezogene Verträge). 
werte für die grundlegende politische Richtung nach dem Souveränitätswechsel festlegt. ${ }^{69}$ Eine Einigung zwischen dem Vereinigten Königreich und der Volksrepublik China war notwendig geworden, da mit Ablauf des 30. Juni 1997 der zwischen dem Vereinigten Königreich und China über die "New Territories" abgeschlossene Pachtvertrag ${ }^{70}$ auslief und die gebietlich wesentlich kleineren Teile Hong Kong Island und Kowloon alleine nicht lebensfähig sind. ${ }^{71}$

Art. 3 Abs. 2 der "Joint Declaration" lautet: "[...] The Hong Kong Special Administrative Region will enjoy a high degree of autonomy, except in foreign and defence affairs which are the responsibilities of the Central People's Government." ${ }^{72}$ Ferner heißt es in Art. 3 Abs. 3 der "Joint Declaration": "The Hong Kong Special Administrative Region will be vested with executive, legislative and independent judicial power, including that of final

Die Rechtsnatur der "Joint Declaration" als verbindlicher völkerrechtlicher Vertrag ist nicht unbestritten. Wie hier Scheuer, M., Die Rechtslage von Hong Kong und Macao nach den "Gemeinsamen Erklärungen" vom 19. Dezember 1984 und 13. April 1987, Fn. 3, S. 48 ff. mit zahlreichen weiteren Nachweisen; Wesley-Smith, P., The SAR Constitution: Law or Politics?, Hong Kong Law Journal, Vol. 27 (1997), Part 2, S. 125 ff. und Luthra, T., Hong Kong: Von der britischen Kronkolonie zur Sonderverwaltungszone in der Volksrepublik China, RIW 1997, S. $625 \mathrm{f}$.

Vertrag zwischen China und dem Vereinigten Königreich, die Ausdehnung des Gebietes von Hong Kong betreffend, vom 6. Juni 1898 ("2. Peking-Vertrag"); vgl. hierzu anschaulich Scheuer, $M$., Die Rechtslage von Hong Kong und Macao nach den "Gemeinsamen Erklärungen" vom 19. Dezember 1984 und 13. April 1987, Fn. 3, S. 24 (30 f.).

Anders als die "New Territories" waren Hong Kong Island und Kowloon nicht gepachtet, sonderm von China an das Vereinigte Königreich abgetreten worden. Mit Bezug auf Hong Kong ist dies in dem Vertrag vom 29. August 1842 zwischen China und dem Vereinigten Königreich ("NankingVertrag") erfolgt, während Kowloon im Vertrag zwischen China und dem Vereinigten Königreich vom 24. Oktober 1860 ("1. Peking-Vertrag") abgetreten wurde. Die Volksrepublik China hat die Verbindlichkeit der vorbezeichneten Verträge und auch die dort vorgenommene Abtretung von Hong Kong und Kowloon durch die Qing-Dynastie nicht anerkannt, sonderm die Verträge stets als "ungleich" bezeichnet. Aus der "Ungleichheit" der Verträge schloß die Volksrepublik China auf deren Unwirksamkeit, aus der sich auch ergebe, daß die Regelung der Hong Kong-Frage zu jeder Zeit souverän und ausschließlich durch die Volksrepublik China gelöst werden könne. Aus dieser Sichtweise erklärt sich auch, daß die Vertreter der Volksrepublik China im Zusammenhang mit der Übergabe Hong Kongs nie den Begriff des Souveränitätswechsels gebraucht, sondem stets von der Wiederausübung der Souveränität über Hong Kong durch die Volksrepublik China gesprochen haben. Die tatsächliche Haltung der Volksrepublik China zum Beispiel zur Vertretung von Hong Kong gegenüber dritten Staaten durch das Vereinigte Königreich (vor dem Souveränitätswechsel) steht jedoch im Gegensatz zu der "offiziellen Haltung". Vgl. mit zahlreichen Nachweisen Scheuer, M., Die Rechtslage von Hong Kong und Macao nach den "Gemeinsamen Erklärungen" vom 19. Dezember 1984 und 13. April 1987, Fn. 3, S. 24 ff.

Die Sonderverwaltungszone Hong Kong wird einen hohen Grad an Autonomie genießen, außer in auswärtigen- und Verteidigungsangelegenheiten, die Verantwortlichkeiten der Zentralen Volksregierung sind. 
adjudication. [...]." ${ }^{73}$ Die weitgehende Autonomie der Sonderverwaltungszone Hong Kong wird zudem in Annex I zur "Joint Declaration" betont. ${ }^{74}$ Die "Joint Declaration", deren Festlegungen gemäß Art. 12 für einen Zeitraum von 50 Jahren nach dem Souveränitätswechsel unverändert bleiben sollen, weist als zentrale Bestimmung neben der Rückgabevereinbarung der Sonderverwaltungszone Hong Kong ein hohes Maß an Autonomie zu, die sich in Exekutiv-, Legislativ- und insbesondere in unabhängigen und abschließenden Judikativkompetenzen ausdrückt. $^{75}$

\section{c) Das "Basic Law"}

Durch die Verabschiedung des "Basic Law" als gesetzlicher Kodifikation hat der Nationale Volkskongreß die der Kronkolonie Hong Kong zuvor unter anderem in der "Joint Declaration" zugesicherte Autonomie verfassungsrechtlich manifestiert. Dabei gestattet Artikel 31 der Verfassung der Volksrepublik China ${ }^{76}$ die Einrichtung von Sonderverwaltungszonen und zugleich die Festlegung des in der betreffenden Sonderverwaltungszone einzurichtenden "Systems". Die Kompetenz für die Entscheidung über die Errichtung von Sonderverwaltungszonen einschließlich der Festlegung des dortigen "Systems" ist in Art. 62 Nr. 13 der Verfassung der Volksrepublik China dem Nationalen Volkskongreß zugewiesen.

Das in der "Joint Declaration" zwischen dem Vereinigten Königreich und der Volksrepublik China vereinbarte Autonomieprinzip wurde vom Nationalen Volkskongreß bei der Ausgestaltung des "Basic Law" berücksichtigt und konkretisiert. ${ }^{77}$ Art. 2 des "Basic Law" lautet: "The National People's Congress authorizes the Hong Kong Special Administrative Region to exercise a high degree of autonomy and enjoy executive, legislative and independent judicial power, including that of final adjudication, in accordance with the provi-

Die Sonderverwaltungszone Hong Kong wird mit Exekutiv-, Legislativ- und unabhängigen Judikativbefugnissen, einschließlich der der letzten richterlichen Entscheidung, ausgestattet sein.

74

Ress, G., "The Legal Status of Hong Kong after 1997", ZaöRV 1986, S. 647 ff. (682 ff.)

Vgl. etwa Cheung, A., From Colony to Special Admistrative Region: Issues of Hong Kong's Autonomy within a Centralized Authoritarianistic State, VRÜ, Bd. 31 (1998), S. 304.

76

Vgl. Cheung, A., From Colony to Special Admistrative Region: Issues of Hong Kong's Autonomy within a Centralized Authoritarianistic State, VRÜ, Bd. 31 (1998), S. 303; Jones, D. in: Folsom, $R$. / Minan, J., Law in the People's Republic of China, Dordrecht / Boston / London 1989, S. 918 ff.

77

Art. 12 der "Joint Declaration" sieht den Erlaß des "Basic Law" vor: "The above-stated basic policies of the People's Republic of China regarding Hong Kong and the elaboration of them in Annex I to this Joint Declaration will be stipulated in a Basic Law of the Hong Kong Special Administrative Region of the People's Republic of China, and they will remain unchanged for 50 years." 
sions of this law." ${ }^{78}$ Detaillierte Regelungen zur Ausgestaltung der Autonomie für die Sonderverwaltungszone Hong Kong finden sich in Kapitel II des "Basic Law". 79

Bei dem "Basic Law" handelt es sich um ein Gesetz mit Verfassungscharakter für die Sonderverwaltungszone Hong Kong. In der diesem Beitrag zugrunde liegenden Urteilsbegründung wird das "Basic Law" von dem vorsitzenden Richter beschrieben als Umsetzung eines internationalen Vertrages, nämlich der "Joint Declarartion", welches darüber hinaus zugleich nationales Recht der Volksrepublik China und Verfassung für die Sonderverwaltungszone Hong Kong sei. ${ }^{80}$ Diese Beurteilung wird von den beiden beisitzenden Richtern bestätigt ${ }^{81}$ und findet Zustimmung im Schrifttum. ${ }^{82}$ Die eminente Bedeutung des "Basic Law" ergibt sich unter anderem aus diesem selbst, wobei zu berücksichtigen ist, daß das "Basic Law" eine gesetzliche Kodifikation des Nationalen Volkskongresses als höchstem Staatsorgan der Volksrepublik China ist. Art. 11 des "Basic Law" bestimmt, daß kein Gesetz, welches durch die Legislative in der Sonderverwaltungszone Hong Kong verabschiedet wird, gültig sein soll, soweit es dem "Basic Law" widerspricht. ${ }^{83}$ Ferner stellt Art. 159 des "Basic Law" erhöhte Anforderungen an seine Abänderung durch den Nationalen Volkskongreß. Zugleich bestimmt diese Vorschrift ausdrücklich, daß keine Änderungen oder Ergänzungen des "Basic Law" zulässig sein sollen, die den grundlegenden politischen

Der Nationale Volkskongreß gestattet der Sonderverwaltungszone Hong Kong ein hohes Maß an Autonomie und unabhängige Exekutiv-, Legislativ- und Judikativbefugnisse, einschließlich der der letzten richterlichen Entscheidung, auszuüben, soweit dies mit den Vorschriften dieses Gesetzes vereinbar ist.

Art. 12 bis Art. 13 des "Basic Law". Vgl. International Legal Materials (ILM), Documents 1990 (Vol. XXIX), S. $1519 \mathrm{ff}$.

Vgl. [1997] 2 HKC S. 315 (323): "The Basic Law is not only a brainchild of an intermational treaty, the Joint Declaration. It is also a national law of the PRC and the constitution of the HKSAR."

Vgl. [1997] 2 HKC S. 315 (345): "It is not in dispute that the primary source of law of the Hong Kong Special Administrative Region of the People's Republic of China ('HKSAR') is the Basic Law enacted by the National People's Congress of the People's Republic of China." und [1997] 2 HKC S. 315 (361) "The Basic Law is Chinese law applicable to the HKSAR and is semi-constitutional."

Wesley'Smith, P., The SAR Constitution: Law or Politics?, Hong Kong Law Joumal, Vol. 27 (1997), Part 2, S. 125 ff., der zugleich auf die weitgehenden Interpretationsbefugnisse, die das "Basic Law" dem Nationalen Volkskongreß gewährt, hinweist. Ferner Scheuer, M., Die Rechtslage von Hong Kong und Macao nach den "Gemeinsamen Erklärungen" vom 19. Dezember 1984 und 13. April 1987, Fn. 3, S. $111 \mathrm{ff}$; $E$, A., Why we must uphold the rule of law, South China Morming Post vom 27. Juli 1997. Kritisch Yash Ghai, Hong Kong's New Constitutional Order, Fn. 4, S. 61 f. und $137 \mathrm{ff}$., der unterschiedliche Ansichten zum Normcharakter des "Basic Law" herausstellt.

Vgl. Yash Ghai, Hong Kong's New Constitutional Order, Fn. 4, S. 337. 
Richtlinien der Volksrepublik China in bezug auf Hong Kong (damit ist insbesondere die "Joint Declaration" gemeint) ${ }^{84}$ zuwiderlaufen.

Durch die Verabschiedung des "Basic Law" hat der Nationale Volkskongreß als oberstes Gesetzgebungsorgan der Volksrepublik China zu erkennen gegeben, daß er sich an die im "Basic Law" getroffenen Regelungen gebunden sieht. Das ergibt sich insbesondere aus der Tatsache, daß in Art. 159 des "Basic Law" Vorschriften enthalten sind, die das Verfahren zur Abänderung durch den Nationalen Volkskongreß festlegen. Einer solchen Kodifikation der Voraussetzungen für eine Abänderung des "Basic Law" hätte es nicht bedurft, wenn der Nationale Volkskongreß im übrigen davon ausgegangen ist, daß die auf ihn zurückführbaren sonstigen Rechtsakte nicht der Überprüfung am Maßstab des "Basic Law" unterliegen. Klarstellend sei insofern darauf hingewiesen, daß der Nationale Volkskongreß die Kompetenz besessen hat, ${ }^{85}$ unter den Voraussetzungen des Art. 159 des "Basic Law" eine Abänderung vorzunehmen. Diesen Weg hat der Nationale Volkskongreß jedoch nicht beschritten, als er das "Preparatory Committee" mandatierte, das "Provisional Legislative Council" einzurichten. ${ }^{86}$ Ebensowenig kam es zu einer Ergänzung oder Abänderung des "Basic Law", welche die durch die Einrichtung des "Provisional Legislative Council" als temporäres Legislativorgan bedingte Abweichung von den Bestimmungen des Art. 68 des "Basic Law" und dessen Annex II legitimieren würde. ${ }^{87}$ Sowohl die Einrichtung und Mandatierung des "Preparatory Committees" durch den Nationalen Volkskongreß bzw. dessen Ständigem Ausschuß, als auch die Einrichtung des "Provisional Legislative Council" erfolgten vielmehr durch einfachgesetzliche Rechtsakte. Jedenfalls die Einrichtung des "Provisional Legislative Council" unterfällt, ebenso wie die Rechtsakte des "Provisional Legislative Council" selbst, dem Prüfungsmaßstab des "Basic Law" und der Jurisdiktionsgewalt der Gerichte in der Sonderverwaltungszone Hong Kong.

In der mit der Verabschiedung des "Basic Law" konstituierten Bindung des Nationalen Volkskongresses an das "Basic Law" liegt zugleich eine Übertragung der Jurisdiktionsgewalt auf die Gerichte in der Sonderverwaltungszone Hong Kong. Das gilt zumindest soweit

84

85

86

87

88

Vgl. Yash Ghai, Hong Kong's New Constitutional Order, Fn. 4, S. 178 f.; Scheuer, M., Die Rechtslage von Hong Kong und Macao nach den "Gemeinsamen Erklärungen" vom 19. Dezember 1984 und 13. April 1987, Fn. 3, S. 146.

Und auch noch besitzt.

Kritisch hierzu auch Wesley-Smith, P., The SAR Constitution: Law or Politics?, Hong Kong Law Joumal, Vol. 27 (1997), Part 2, S. 125 ff. (126).

Vgl. hierzu oben IV.

Das "Basic Law" trifft keine Bestimmungen zum "Preparatory Committee", verweist in dessen Annex II allerdings auf die Entscheidung des Nationalen Volkskongresses vom 4. April 1990, mit welcher die Gründung des "Preparatory Committees" im Jahr 1996 beschlossen wird. Vgl. hierzu Yash Ghai, Hong Kong's New Constitutional Order, Fn. 4, S. 571. 
es um die Überprüfung solcher Rechtsakte geht, die auf den Nationalen Volkskongreß zurückzuführen sind und ausschließlich Wirksamkeit in der Sonderverwaltungszone Hong Kong entfalten. Ein Hinweis für die Richtigkeit dieser Ansicht findet sich im "Basic Law" selbst, in dessen Art. 19 der Sonderverwaltungszone Hong Kong das Recht unabhängiger und abschließender Jurisdiktionsgewalt zugesprochen wird. ${ }^{89}$ Zugleich sollen nach Art. 19 Abs. 2 des "Basic Law" die Grenzen der Rechtsprechungsgewalt aus dem vor dem Souveränitätswechsel in Hong Kong gültigen Rechtssystem bestehen bleiben. ${ }^{90}$ Art. 19 Abs. 2 des "Basic Law" ist auslegungsbedürftig, da es das "Basic Law" als rechtswirksame Kodifikation vor dem Souveränitätswechsel in Hong Kong gerade nicht gab. Insofern kann Art. 19 Abs. 2 des "Basic Law" die Rechtsprechungsgewalt von Gerichten in der Sonderverwaltungszone Hong Kong nicht beschränken, soweit Prüfungsmaßstab im Verfahren vor den Gerichten in der Sonderverwaltungszone Hong Kong das "Basic Law" selbst ist. Der vom vorsitzenden Richter in der Urteilsbegründung angeführte Vergleich zur Beschränkung der Jurisdiktionsgewalt der Gerichte in Hong Kong in bezug auf die Überprüfung des "Letters Patent" und der "Royal Instructions" Law" nicht an die Stelle des "Letters Patent" und der "Royal Instructions" getreten ist, sondern im Vergleich zu jenen ein "Aliud" darstellt. ${ }^{92}$ Das ergibt sich bereits daraus, daß Hong Kong vor dem Souveränitätswechsel Kolonie war, während die Sonderverwaltungszone Hong Kong mit autonomen Rechten ausgestatteter Teil der Volksrepublik China ist. Beschränkt Art. 19 Abs. 2 des "Basic Law" die Jurisdiktionsgewalt der Gerichte in der Sonderverwaltungszone Hong Kong insoweit nicht, so ergibt sich ein positives Indiz für den Umfang der Rechtsprechungsgewalt aus Art. 19 Abs. 3 des "Basic Law", mit welchem

Art. 19 Abs. 1 des "Basic Law" lautet: "The Hong Kong Special Administrative Region shall be vested with independent judicial power, including that of final adjudication."

Art. 19 Abs. 3 des "Basic Law" lautet: "The courts of the Hong Kong Special Administrative Region shall have jurisdiction over all cases in the Region, except that the restrictions on their jurisdiction imposed by the legal system and principles previously in force in Hong Kong shall be maintained."

Vgl. [1997] 2 HKC S. 315 (334 ff.). Der "Letters Patent" und die "Royal Instructions" waren die Rechtsgrundlage, aus welcher der Gouvermeur von Hong Kong seine Legitimation ableitete. Vgl. zum "Letters Patent" und den "Royal Instructions" Finnis in: Lord Hailsham of St. Marylebone (Editor in Chief), Halsbury's Laws of England, Vol. 6, 4. Aufl. 1991, Para. 1060; sowie Scheuer, $M$., Die Rechtslage von Hong Kong und Macao nach den "Gemeinsamen Erklärungen" vom 19. Dezember 1984 und 13. April 1987, Fn. 3, S. 15 ff.

So auch Choy. L., Self-imposed limits could sacrifice autonomy, law academic wams, die Prof. Chan zitiert: "The court should not equate the situation of the SAR to that of the colonial era when Hong Kong had no say over British Parliament's acts. The difference was that the SAR now had its own constitution, Mr. Chan said." So auch Yash Ghai, Dark day for our rights, South China Morning Post vom 30. Juli 1997, der ausführt: "As with the previous point, the court completely disregarded fundamental differences in the constitutional status of Hong Kong before July 1 , 1997, and now. Then Hong Kong was a colony, fully subject to its colonial masters. The SAR is part of China, not its colony. The relationship between them is delineated in great detail in the Basic Law (following the Joint Declaration)." 
die Bereiche Verteidigung und auswärtige Beziehungen ausdrücklich von der Rechtsprechungsgewalt lokaler Gerichte ausgenommen werden. ${ }^{93}$ Im Umkehrschluß ergibt sich, daß für Rechtsakte des Nationalen Volkskongresses, die nicht den beiden genannten Bereichen zuzurechnen sind, den Gerichten in der Sonderverwaltungszone Hong Kong unbeschränkte Rechtsprechungsgewalt zukommt.

Aus der verfassungsrechtlichen Bedeutung folgt, daß den Gerichten in der Sonderverwaltungszone Hong Kong die Kompetenz zustehen muß, alle Rechtsakte, die in der Normenhierarchie unter dem "Basic Law" stehen, am Maßstab des "Basic Law" zu überprüfen. ${ }^{94}$ Diese Kompetenz besteht unabhängig davon, ob der zu prüfende Rechtsakt von einem Legislativorgan in der Sonderverwaltungszone Hong Kong oder von einem sonstigen Legislativorgan der Volksrepublik China, etwa dem Nationalen Volkskongreß oder dessen Ständigem Ausschuß, stammt. Dabei bleibt die endgültige Beurteilung des staatsrechtlichen Verhältnisses zwischen der Volksrepublik China und der Sonderverwaltungszone Hong Kong ohne Auswirkung auf den Umfang der Jurisdiktionsgewalt von Gerichten in der Sonderverwaltungszone Hong Kong. ${ }^{95}$ Eine von dieser Ansicht abweichende Interpretation würde das "Basic Law" tatsächlich zu einer rein deklaratorischen Absichtserklärung herabstufen. ${ }^{96}$

Art. 19 Abs. 3 des "Basic Law" lautet: "The courts of the Hong Kong Special Administrative Region shall have no jurisdiction over acts of state such as defence and foreign affairs. [...]". Ausführlich hierzu auch Cheung, A., From Colony to Special Admistrative Region: Issues of Hong Kong's Autonomy within a Centralized Authoritarianistic State, VRÜ, Bd. 31 (1998), S. 302 ff.

Ob darüber hinaus auch den Gerichten in der Volksrepublik China eine Rechtsprechungsgewalt bezüglich dieser Frage zukommt, soll an dieser Stelle nicht erörtert werden. Vgl. hierzu unten V. 3. d).

Zu den unterschiedlichen Formen föderalistischer, autonomer und dezentraler Gebietsstrukturen und deren Bedeutung für das staatsrechtliche Verhältnis der Volksrepublik China und der Sonderverwaltungszone Hong Kong vgl. Yash Ghai, Hong Kong's New Constitutional Order, Fn. 4, S. $179 \mathrm{ff}$.

So auch mit Bezug auf die Sichtweise der Funktionäre und Ideologen in der Volksrepublik China Wesley-Smith, P., The SAR Constitution: Law or Politics?, Hong Kong Law Joumal, Vol. 27 (1997), Part 2, S. 125 (126) der ausführt: "For China's functionaries and ideologues, therefore, neither the Joint Declaration nor the Basic Law can be taken at face value. In an ultimate sense they are not legal documents at all but political manifestos, not necessarily any more enduring than party platforms in an election campaign." Vgl. auch Choy, L. / Ku, G., Fears as 'bulwark of Basic Law falls', South China Morning Post vom 30 Juli 1997. Ferner Yash Ghai, Dark day for our rights, South China Moming Post vom 30. Juli 1997, der anmerkt: "It is hard to imagine a more disastrous judgement for the future of the doctrine of 'one country two systems' and the high degree of autonomy of the Hong Kong Special Administrative Region than the one given yesterday by the Court of Appeal." 


\section{d) Die Rechtsprechungsgewalt der Gerichte in der Volksrepublik China}

Der Umfang der Jurisdiktionskompetenz von Gerichten in der Sonderverwaltungszone Hong Kong ergibt sich zudem aus einer weiteren Überlegung: Verneint man die Kompetenz der Gerichte in der Sonderverwaltungszone Hong Kong, Rechtsakte des Nationalen Volkskongresses, die sich auf die Sonderverwaltungszone Hong Kong beziehen, am Maßstab des "Basic Law" zu überprüfen, so muß diese Kompetenz zumindest einem Gericht in der Volksrepublik China zustehen. Ein solches gerichtliches Verfahren müßte zudem von einer Behörde in der Sonderverwaltungszone Hong Kong eingeleitet werden können. Soweit ein derartiges Verfahren vor einem Gericht in der Volksrepublik China nicht eingeleitet werden könnte, bestünde keine Möglichkeit zur Überprüfung von Rechtsakten des Nationalen Volkskongresses, die ihre Wirksamkeit in der Sonderverwaltungszone Hong Kong entfalten. ${ }^{97}$ Das würde nicht nur den Kernbereich rechtsstaatlicher Prinzipien verletzen, sondern auch die Kodifikation des "Basic Law" und die dort und in der "Joint Declaration" festgeschriebene Autonomie der Sonderverwaltungszone Hong Kong absurd machen. Denn wenn keiner Instanz die Kompetenz zur Überprüfung von Rechtsakten des Nationalen Volkskongresses am Maßstab des "Basic Law" zukommt, bliebe das "Basic Law" insofern anwendungslos. In diesem Fall wäre der Nationale Volkskongreß bei der Verabschiedung Hong Kong bezogener Rechtsakte keinerlei nachprüfbarer Bindung unterworfen und könnte daher sogar willkürlich handeln. Ein solches Verständnis widerspricht jedoch der verfassungsrechtlichen Bedeutung des "Basic Law" und würde die der Sonderverwaltungszone Hong Kong zugebilligte Autonomie vollständig aushöhlen.

Bestimmungen zur Rechtsprechung finden sich in der Verfassung der Volksrepublik China im 7. Abschnitt des 3. Kapitels (Art. 123 - Art. 135). ${ }^{98}$ Art. 123 der Verfassung der Volksrepublik China bezeichnet die Volksgerichte als Organe der Rechtsprechung in der Volksrepublik China, wobei in den folgenden Artikeln das Oberste Volksgericht als höchstes Organ der Rechtsprechung festgelegt wird. ${ }^{99}$ Die Verfassung der Volksrepublik China trifft demgegenüber selbst keine Bestimmung zu den zulässigen Verfahrensarten vor dem Obersten Volksgericht und den Volksgerichten. ${ }^{100}$ Bemerkenswert ist auch, daß den Volksgerichten, einschließlich dem Obersten Volksgericht, nicht die Kompetenz zukommt, die

Cheung, A., From Colony to Special Admistrative Region: Issues of Hong Kong's Autonomy within a Centralized Authoritarianistic State, VRÜ, Bd. 31 (1998), S. 310.

98 Fundstellen vgl. oben Fn. 55.

99 Vgl. Art. 124 und Art. 127 der Verfassung der Volksrepublik China. Fundstellen vgl. oben Fn. 55. 100

Von einer Unabhängigkeit der Rechtsprechung in der Volksrepublik China ist jedoch schon unter Berücksichtigung von Art. 128 der Verfassung der Volksrepublik China nicht auszugehen, der bestimmt, daß das Oberste Volksgericht dem Nationalen Volkskongreß und dessen Ständigem Ausschuß verantwortlich ist, während die lokalen Volksgerichte aller Ebenen den Organen der Staatsmacht, die sie errichten, verantwortlich sind. 
Verfassung der Volksrepublik China oder sonstiges Gesetzesrecht zu interpretieren. ${ }^{101}$ Dieses Recht steht vielmehr dem Nationalen Volkskongreß und dessen Ständigem Ausschuß zu. ${ }^{102}$ Auch das Gerichtsorganisationsgesetz von $1979^{103}$ enthält keine Regelung, die einem der Gerichte in der Volksrepublik China eine Kompetenz in verfassungsrechtlichen Streitigkeiten zuweist. Gleichzeitig fehlt es im Verhältnis der Volksrepublik China und der Sonderverwaltungszone Hong Kong ganz allgemein an der Festlegung einer Verfahrensart oder nur einem Mechanismus, nach welchem Meinungsverschiedenheiten über die Interpretation des "Basic Law" oder über die Rechtsbeziehungen zueinander einer rechtlichen Klärung unterzogen werden können. ${ }^{104}$ Aus dieser Rechtslage ergibt sich, daß tatsächlich weder nach dem Verfassungsrecht noch nach dem Gerichtsorganisationsrecht der Volksrepublik China eine Verfahrensart vorgesehen ist, mit der Rechtsakte des Nationalen Volkskongresses mit Bezug auf Hong Kong von den Gerichten in der Volksrepublik China am Maßstab des "Basic Law" überprüft werden könnten. ${ }^{105}$ Konsequenterweise stellt sich die weitere Frage nach einem Initiativrecht von Behörden in der Sonderverwaltungszone Hong Kong zur Einleitung eines diesbezüglichen Verfahrens erst gar nicht. Zugleich ist es perplex, die Kompetenz für die verbindliche Überprüfung von Hong Kong-bezogenen Rechtsakten dem Nationalen Volkskongreß beziehungsweise dessen Ständigem Ausschuß zuzubilligen. ${ }^{106}$ Da der Nationale Volkskongreß das Legislativorgan der Volksrepublik China ist, würde der Nationale Volkskongreß abschließend über die Verfassungs- und "Basic Law"-Konformität seiner eigenen Rechtsakte entscheiden. Ein derartiges Ergebnis steht im Widerspruch zu den Grundsätzen der "Joint Declaration" und des "Basic Law", nach denen das bisher in Hong Kong geltende Rechtssystem und die bisherigen Rechtsgrundsätze, zu denen auch das Prinzip der Gewaltenteilung gehört, grundsätzlich fortbestehen sollen. ${ }^{107}$

Aus der fehlenden gerichtlichen Überprüfungsmöglichkeit von Rechtsakten des Nationalen Volkskongresses am Maßstab des "Basic Law" vor Gerichten in der Volksrepublik China

101

Folsom, R. / Minan, J., Law in the People's Republic of China, Fn. 74, S. 170. Differenzierend Senger, H. von, Einführung in das chinesische Recht, München 1994, S. $180 \mathrm{ff}$.

Folsom, R. / Minan, J., Law in the People's Republic of China, Fn. 74, S. 170.

103 "Organic Law of the People's Courts". Der Text ist in englischer Sprache abgedruckt bei Folsom, 104 R. / Minan, J., Law in the People's Republic of China, Fn. 74, S. $174 \mathrm{ff}$.

Im einzelnen hierzu Cheung, A., From Colony to Special Admistrative Region: Issues of Hong Kong's Autonomy within a Centralized Authoritarianistic State, VRÜ, Bd. 31 (1998), S. 310; Yash Ghai, Hong Kong's New Constitutional Order, Fn. 4, S. 183.

Cheung, A., From Colony to Special Admistrative Region: Issues of Hong Kong's Autonomy within a Centralized Authoritarianistic State, VRÜ, Bd. 31 (1998), S. 310.

Kritisch hierzu auch Wesley-Smith, P., The SAR Constitution: Law or Politics, Hong Kong Law 107 Journal, Vol. 27 (1997), Part 2, S. 125 ff.

Vgl. oben V. 3. b) und V. 3. c). 
wird deutlich, daß diese Kompetenz den Gerichten in der Sonderverwaltungszone zukommt, da das "Basic Law" anderweitig "leerlaufen" würde. ${ }^{108}$

\section{Zur Rechtmäßigkeit der Einrichtung des "Provisional Legislative Council"}

Die von dem "Court of Appeal" ebenfalls als "obiter dicta" getroffenen Feststellungen zur Rechtmäßigkeit des "Provisional Legislative Council" ${ }^{109}$ sind in rechtlicher Hinsicht unzutreffend und verdienen daher keine Zustimmung. ${ }^{10}$ Das Verfahren zur Einrichtung des "Legislative Council", welches nach Art. 66 des "Basic Law" das Gesetzgebungsorgan der Sonderverwaltungszone Hong Kong sein soll, ist in Art. 68 des "Basic Law" geregelt. Art. 68 Satz 1 des "Basic Law" stellt sicher, daß das "Legislative Council" durch Wahlen zustande kommt. Dabei konkretisiert Art. 68 Satz 2 des "Basic Law", daß die Methode zur Einrichtung des "Legislative Council" vor dem Hintergrund der jeweiligen Situation festgelegt werden soll, wobei ein allmählicher Fortschritt mit dem Ziel der Wahl aller Abgeordneten durch allgemeine Wahlen angestrebt werden soll. Schließlich verweist Art. 68 Satz 3 des "Basic Law" auf dessn Annex II, in welchem detaillierte Regelungen zur Wahl des "Legislative Council" getroffen sind. ${ }^{11}$ Immerhin ist dem "Court of Appeal" zuzustimmen, daß Annex II zum "Basic Law" keine Regelungen bezüglich des ersten "Legislative Council" der Sonderverwaltungszone Hong Kong enthält. Die in Annex II zum "Basic Law" getroffene Unterteilung in Mandatsträger aus "functional constituencies", dem "Election Committee" und "geographical constituencies" soll gerade nicht für das erste "Legislative Council" gelten. Die Kompetenz zur Festlegung des Verfahrens für das erste "Legislative Council" wird in Annex II zum "Basic Law" dem Nationalen Volkskongreß zugewiesen.

108

So auch Wesley-Smith, P., The SAR Constitution: Law or Politics?, Hong Kong Law Joumal, Vol. 27 (1997), Part 2, S. 125 ff.

109 Zum geschichtlichen Hintergrund und zum Verfahren bei der Einrichtung des "Provisional Legislative Council" durch den Nationalen Volkskongreß und das "Preparatory Committee" vgl. oben I. und ausführlich Chen, A., The Provisional Legislative Council of the SAR, Hong Kong Law Journal, Vol. 27 (1997), Part 1, S. 1 ff.

110 Vgl. die Ausführungen des vorsitzenden Richters [1997] 2 HKC S. 315 (335 ff.)

111 Art. 68 des "Basic Law" lautet: "The Legislative Council of the Hong Kong Special Administrative Region shall be constituted by election. The method for forming the Legislative Council shall be specified in the light of the actual situation in the Hong Kong Special Administrative Region and in accordance with the principle of gradual and orderly progress. The ultimate aim is the election of all the members of the Legislative Council by universal suffrage. The specific method for the Formation of the Legislative Council and its procedures for voting on bills and motions are prescribed in Annex II: 'Method for the formation of the Legislative Council of the Hong Kong Special Administrative Region and Its Voting Procedures'." 
Aus der Tatsache, daß das erste "Legislative Council" von der detaillierten Regelung des Wahlverfahrens in Annex II zum "Basic Law" ausgenommen ist, läßt sich jedoch nicht folgern, daß der Nationale Volkskongreß bei der Ausgestaltung des Wahlverfahrens für das erste "Legislative Council" vollkommen frei wäre. Die dahingehende Auffassung des "Court of Appeal" ist unzutreffend. ${ }^{112}$ Richtigerweise ist vielmehr von einer Bindung des Nationalen Volkskongresses an die allgemeinen Wahlgrundsätze aus Art. 68 Satz 1 und Satz 2 des "Basic Law" auch bei der Verfahrensregelung zur Einrichtung des ersten "Legislative Council" auszugehen. Das bestätigt sich bereits aus Art. 69 des "Basic Law", der die Amtszeit des ersten "Legislativ Council" auf zwei Jahre begrenzt, während die Wahlperiode für die darauf folgende Zeit auf vier Jahre festgelegt ist. Aus dieser Vorschrift ergibt sich, daß die Regelungen des "Basic Law" grundsätzlich auch auf das erste "Legislative Council" anwendbar sein sollen. Eine Ausnahme besteht nur bezüglich Annex II zum "Basic Law". Wäre demgegenüber die Unanwendbarkeit des gesamten "Basic Law" in Bezug auf das erste "Legislativ Council" beabsichtigt gewesen, so hätte eine diesbezügliche Ausnahme systematisch nicht in Annex II zum "Basic Law" normiert werden dürfen, sondern im Hauptteil des "Basic Law" selbst, mithin in Artikel 66 ff. Daß dies aber gerade nicht der Fall sein sollte, zeigt sich, wie bereits ausgeführt, an Art. 69 des "Basic Law". Das Urteil ist in diesem Bereich also schon deshalb unrichtig, weil es eine Bindung des Nationalen Volkskongresses an das "Basic Law" bei der Einrichtung des ersten "Legislative Council" unzutreffend verneint.

Unverständlich ist die weitere Begründung, die darauf abstellt, daß es sich bei dem "Provisional Legislative Council" im übrigen gar nicht um das erste "Legislativ Council" im Sinne des "Basic Law" handeln soll. ${ }^{113}$ Die Argumentation des "Court of Appeal", daß das "Provisional Legislative Council" ein übergangsweise bestehendes Legislativorgan sei, welches die erste Regierung der Sonderverwaltungszone Hong Kong bei den Vorbereitungen zur Einrichtung des ersten "Legislative Council" im Sinne des "Basic Law" unterstütze, entbehrt jeder rechtlichen Grundlage. An keiner Stelle im "Basic Law" findet sich ein Hinweis auf ein solches übergangsweise einzurichtendes Legislativorgan. ${ }^{114}$ Vielmehr bestätigen die Hinweise auf das erste "Legislative Council" in Art. 69 des "Basic Law" und

\footnotetext{
112 Vgl. [1997] 2 HKC S. 315 (340 f.).

$113 \mathrm{Vgl}$. [1997] 2 HKC S. 315 (341 f.).

114 So - zumindest die fehlende Legitimation des "Provisional Legislative Council" durch das "Basic Law" betreffend - auch Chen, A., The provisional legislative Council of the SAR, Hong Kong Law Journal, Vol. 27 (1997), Part 1, S 1 ff. (S. 7 f.), der ausführlich auf die Kontroverse zwischen dem Vereinigten Königreich und der Volksrepublik China um die Einrichtung des ersten Legislativorgans für die Sonderverwaltungszone Hong Kong eingeht. Die Legitimation des "Provisional Legislative Council" bezweif elnd Lo Shiu-hing, Hong Kong's Legislative Council as a Symbol of Political Development, Hong Kong Law Joumal, Vol. 27 (1997), Part 2, S. 271 ff. Vgl. auch Pearsons, C., Interim body 'brakes rules for first SAR legislature', South China Moming Post vom 24. Juli 1997.
} 
in dessen Annex II, daß bei der Verabschiedung des "Basic Law" von der nahtlosen Einrichtung und Arbeitsaufnahme des ersten "Legislative Council" ab dem Zeitpunkt des Souveränitätswechsels ausgegangen wurde. Für die Einrichtung eines vorgeschalteten, übergangsweise tätigen "provisorischen" Gesetzgebungsorgans läßt das "Basic Law" somit keinen Raum. Auch bei der Einrichtung des "Provisional Legislative Council" als "provisorischem" Gesetzgebungsorgan hätte der Nationale Volkskongreß vor dessen Einrichtung das "Basic Law" entsprechend abändern müssen. Eine diesbezügliche Anpassung unterblieb jedoch. ${ }^{115}$

Die Einrichtung des "Provisional Legislative Council" verstößt gegen Art. 68 des "Basic Law" und ist damit rechtswidrig. Dieser Verstoß wurde auch von dem Vertreter der Anklage zugestanden. ${ }^{116}$ Wegen der verfassungsrechtlichen Bedeutung des "Basic Law" führt der Verstoß bei der Einrichtung des "Provisional Legislative Council" auch zu dessen Nichtigkeit. Das gegenteilige Ergebnis, zu dem das zitierte Urteil kommt, ist willkürlich; die Bezeichnung des "Provisional Legislative Council" als vorübergehendes Legislativorgan, auf das die Bestimmungen des "Basic Law" schlicht keine Anwendung finden sollen, stellt einen unzulässigen Kunstgriff dar.

\section{Zusammenfassung}

Durch die Rechtssache der Sonderverwaltungszone Hong Kong gegen David Ma und andere hatte der "Court of Appeal" in der Zeit unmittelbar nach dem Souveränitätswechsel Gelegenheit, die der Sonderverwaltungszone Hong Kong in der "Joint Declaration" und dem "Basic Law" zugesicherte Autonomie unter dem Grundsatz "One Country Two Systems" zu konturieren und damit die Zusicherung "auf dem Papier" zur Wirklichkeit werden zu lassen. Der "Court of Appeal" hat diese Gelegenheit nicht nur verstreichen lassen, sondern durch die eigene Beschränkung der Jurisdiktionsgewalt und die nicht überzeugende Bestätigung der rechtmäßigen Errichtung des "Provisional Legislative Council" die Bindung des Nationalen Volkskongresses an das "Basic Law" verneint. Damit hat der "Court of Appeal" zugleich dem Nationalen Volkskongreß jeden Freiraum zugebilligt, auch solche Rechtsakte mit Bezug auf die Sonderverwaltungszone Hong Kong zu erlassen, die in klarem Widerspruch zum "Basic Law" stehen, ohne daß die Rechtswidrigkeit solcher Rechtsakte von einem Gericht zwingend festgestellt werden könnte.

115 Die Verteidigung hat auf die Notwendigkeit einer Abänderung des "Basic Law" zur Legitimation des "Provisional Legislative Council" hingewiesen. Vgl. [1997] 2 HKC S. 315 (340). Der vorsitzende Richter führt dazu in seiner Urteilsbegründung jedoch aus: "The NPC could of course amend the Basic Law before it came into effect. But that would be impracticable and politically undesireable." Vgl. [1997] 2 HKC S. 315 (340).

116 Vgl. [1997] 2 HKC S. 315 (341) und Pearsons, C., Law 'had to be broken', South China Morning Post vom 25. Juli 1997. 
Hoffnung für eine Korrektur dieser - für die Autonomie der Sonderverwaltungszone Hong Kong abträglichen - Rechtsprechung des "Court of Appeal" ergibt sich unter zwei Gesichtspunkten. Zum einen erfolgten die Ausführungen des "Court of Appeal" sowohl zur Rechtsprechungsgewalt der Gerichte in der Sonderverwaltungszone Hong Kong als auch zur rechtmäßigen Einrichtung des "Provisional Legislative Council" als "obiter dicta" und damit ohne Bindungswirkung. Zum anderen ist die Entscheidung des "Court of Appeal" nicht letztinstanzlich, da das höchste Gericht in der Sonderverwaltungszone Hong Kong der "Court of Final Appeal" ist. Dieses oberste Gericht in der Sonderverwaltungszone Hong Kong hat bisher zu keiner der vom "Court of Appeal" entschiedenen Fragen Stellung bezogen. 


\section{The Hong Kong Special Administrative Region and Common Law}

\section{By Tim G. Luthra}

With effect from 1st of July 1997 Hong Kong ceased to be a colony of the British Crown and the sovereignty of the People's Republic of China over Hong Kong Special Administrative Region (Hong Kong SAR) was fully restored. Under the Joint Declaration of the Government of the United Kingdom and the People's Republic of China as well as the Basic Law - the two documents that built the framework for the handover and for the administration of Hong Kong SAR in the period after the handover - Hong Kong SAR would "enjoy a high degree of autonomy". The documents referred to provide for the continuous application of all laws previously in force in Hong Kong, that is the common law, rules of equity, ordinances, etc., and the judicial power in Hong Kong SAR to be vested in the courts of Hong Kong SAR which shall exercise their judicial power - including that of final adjudication - independently and free from any interference. The implementation and interpretation of the Joint Declaration and the Basic Law after the handover by authorities both in the People's Republic of China and in Hong Kong SAR has led to great controversy. It is therefore a surprise that the "Reservation of Question of Law No. 1 of 1997 between Hong Kong SAR (Applicant) and Ma Wai-Kwan, David and others (Respondants)" before the Court of Appeal of the High Court and the judgement delivered has not received appropriate response outside Hong Kong SAR.

With the above mentioned judgement the High Court declares its position with respect to the survival of the common law in Hong Kong SAR, the jurisdiction of Hong Kong SAR courts and the legality of the Provisional Legislative Council. Considering the historical context, the wording and the intention of the relevant provisions of the Joint Declaration and the Basic Law, the High Court held that the common law had "automatically" been adopted under the Basic Law itself with no "overt" or "formal" act of adoption required. Therefore the further comments of the High Court on the scope and limits of the jurisdiction of courts within Hong Kong SAR and the legality of the Provisional Legislative Council are obiter. However it is the High Court's statements to these two issues that reveal its interpretation of the autonomy granted to Hong Kong SAR under the Joint Declaration and the Basic Law to be severly limited. The above mentioned judgement denies jurisdiction of the courts in Hong Kong SAR over any decision or resolution (or the reasons behind them) by the National People's Congress as the "highest organ of state power". With respect to the legality of the Provisional Legislative Council the jurisdiction of the courts in Hong Kong SAR, according to the referred judgement, is restricted to examining whether there was any decision or resolution setting up the Provisional Legislative Council and whether in fact this decision or resolution was carried out. The judgement is affirmative with regard to both.

The judgement of the High Court deserves consent as to the automatic adoption of the common law under the Joint Declaration and the Basic Law with no further explicit act of 
adoption being required. On the contrary the obiter on the legality of the Provisional Legislative Council and particularly on the limited scope of the jurisdiction of the courts in Hong Kong SAR have to be examined critically. This part of the judgement is not only doubtful under the issues of the law but also has a severe factual impact to the interpretation of the principle: "One Country Two Systems". Contrary to the position of the High Court, Art. 3 (3) of the Joint Declaration and Arts. 2, 19 and 81 of the Basic Law provide for the unlimited jurisdiction of the courts in Hong Kong SAR with respect to all decisions or resolutions concerning Hong Kong SAR. In adopting and promulgating the Basic Law as national law of the People's Republic of China and - at the same time - as the Constitution of Hong Kong SAR, the People's Republic of China agreed to observe the principles laid down in the Basic Law. This obligation to adhere to the Basic Law would be of no effect if the authorities in the People's Republic of China were to deviate from the principals of the Basic Law with no possibility of challenging such an infringement in a court in Hong Kong SAR. Moreover under this interpretation it would be unreasonable to even think of an independent judiciary within Hong Kong SAR, which would again be a violation of the explicit sections in the Joint Declaration and the Basic Law. Additionally it is doubtful, whether any act of the National People's Congress with regard to Hong Kong SAR could be challenged before a court in the People's Republic of China and if so, whether any such assessment would be that of an independent judiciary.

Hope for a reversal of this restrictive interpretation of Hong Kong SAR's autonomy under the Joint Declaration and the Basic Law by the High Court derives from the two facts that the relevant part of the judgement is obiter and not in the last instance. Hopefully the Court of Final Appeal - if conf ronted with the issues - will take a different position.

\section{Rules and Regulations of the Federal National Council of the United Arab Emirates and the National Consultative Council of the Emirate of Abu Dhabi and their Functions within the Country's Socio-political Sphere}

\section{By Monika Fatima Mühlböck}

On 2 December 1971 six of the seven Trucial states, Abu Dhabi, Dubai, Sharjah, Ajman, Umm al-Qaiwain, and Fujairah formed the United Arab Emirates. The seventh state, Ras alKhaimah, joined the federation on 10 February 1972.

The rulers of the sheikhdoms decided it was necessary to create modern forms of administration including direct democracy, in the form of the Federal National Council and the National Consultative Council, while at the same time preserving traditional institutions like the madjlis, with the vitality and legitimacy they draw from history. The Federal National Council, which deals on a country-wide level, was formally established on 13 